\title{
White Dwarf Remnants of Binary Star Evolution
}

\author{
Christopher A. Tout \\ Institute of Astronomy, The Observatories, Madingley Road, Cambridge CB3 0HA, England \\ email: cat@ast.cam.ac.uk
}

\begin{abstract}
White dwarfs grow as the cores of red giants and, in particular, carbon-oxygen white dwarfs grow in asymptotic giant branch (AGB) stars. The evolution of an AGB star is a competition between growth of the core and loss of the stellar envelope, typically in a wind. It is complicated by thermal pulses driven periodically by unstable helium shell burning. Dredge up following such pulses delays core growth. The compression at the center of a cold carbon-oxygen core means that carbon ignites when it reaches a mass of $1.38 M_{\odot}$. This begins the familiar thermonuclear runaway of the Type Ia supernova (SN Ia). At higher temperatures carbon can ignite more gently and burn mostly to neon to leave a core rich in oxygen, neon and magnesium. Such cores can go on to collapse to neutron stars with a release of only neutrinos. Accepted mass-loss prescriptions for giants mean that the range of masses of single stars that leave carbonoxygen white dwarfs is somewhere from around 1 to $8 M_{\odot}$. We investigate how unusual mass loss, perhaps brought about by interaction with a binary companion, can radically alter the single star picture. Though population syntheses treat some possibilities with various prescriptions, there is sufficient doubt over the physics, the observations, and the implementation of mass loss and binary interaction that there is scope for several more unusual progenitors of carbon-oxygen white dwarfs and hence SNe Ia.
\end{abstract}

Keywords. stars: AGB and post-AGB, mass loss, white dwarfs, supernovae

The physics of stars is complex but today we have a good quantitative grasp of most of what is needed. Indeed we can make highly accurate models of the Sun that have been tested with detailed helioseismology. As stars evolve, both our understanding and ability to test our models decreases. Least well-modeled is perhaps convection, where what goes on in the Sun is extrapolated to all sorts of very different stars, including the deep convective envelopes of giants. Nevertheless, because most of a convective region is very close to adiabatic, our errors are not gross. Our greatest challenge is more the atmosphere and particularly a star's ability to lose mass. We know that the brightest giants lose mass at extremely high rates but we have no physical model that predicts this rate or indeed allows it to be extrapolated to other giants. Similarly we know that binary stars must interact by mass transfer once they are large enough relative to their orbit but how this mass transfer proceeds in many cases is simply guessed at.

\section{The Origin of White Dwarfs}

Stars like the Sun on the main sequence are fusing hydrogen to helium in their cores. In the Sun the energy escapes radiatively in the interior and convectively through the outer layers. At higher masses the burning drives a convective core and the surface is hot enough to prevent convection there. This typical structure for, a $5 M_{\odot}$, main-sequence star is shown in Figure 1A. Once hydrogen is exhausted in the core, burning moves 

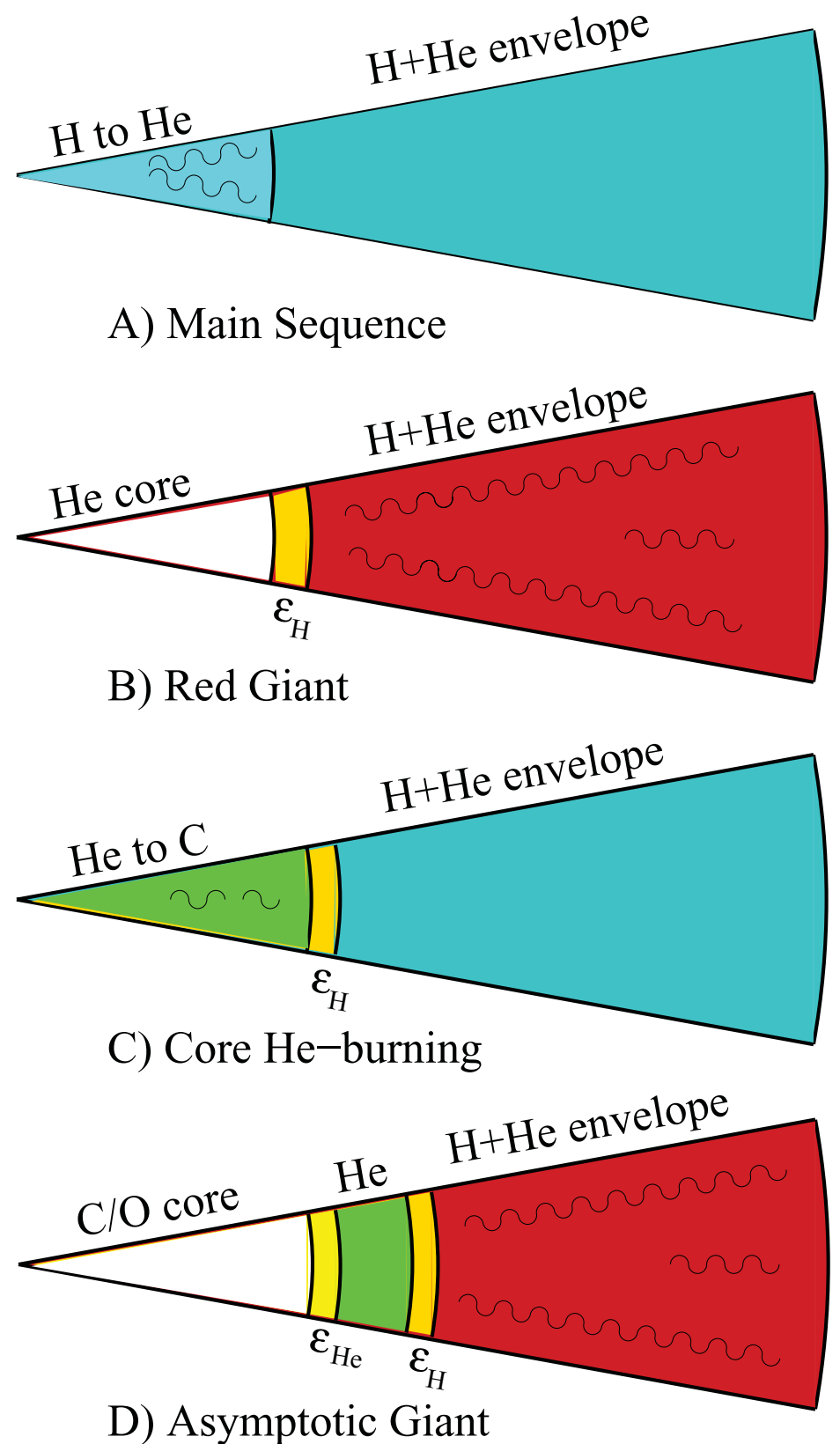

Figure 1. Schematic evolution of the interior of a $5 M_{\odot}$ star. Wavy lines indicate convection. Thin shells are labeled $\epsilon_{\mathrm{H}}$ if burning hydrogen and $\epsilon_{\mathrm{He}}$ if burning helium.

to a shell beyond the helium ash. The star expands and reddens, developing a deep convective envelope. As it proceeds, the star grows a helium core (Figure 1B). If the star were to lose its hydrogen envelope at this point it would leave a helium white dwarf remnant.

As the core grows in mass it contracts in radius and heats up. This raises the temperature at the burning shell so that the reactions run faster and the luminosity rises. The core, growing in mass but contracting in radius, continues to heat up until at $1.2 \times 10^{8} \mathrm{~K}$ 


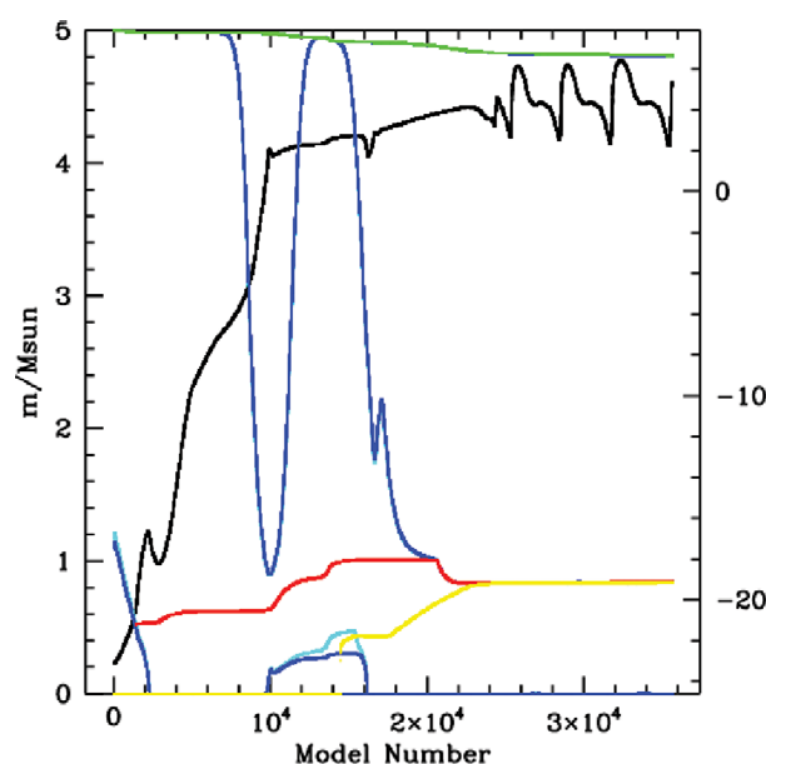

Figure 2. The internal evolution of a $5 M_{\odot}$ star. Time increases monotonically with model number but very non-linearly because more models must be made when the star is changing its structure more rapidly. The darkest line is the helium luminosity as $\log _{10}\left(L_{\mathrm{He}} / \mathrm{erg} \mathrm{s}^{-1}\right)$ on the right-hand axis. Helium burning is an important source of energy only once this has risen above zero. The pale line monotonically slightly decreasing from $5 M_{\odot}$ is the total mass of the star reduced by a standard stellar wind. The remaining dark lines are convective boundaries. At model zero the convective core is visible. Hydrogen shell burning is indicated by the medium line that comes into being just above $0.5 M_{\odot}$ just before the convective core vanishes. Soon after this the convective envelope deepens and then helium burning begins in the core again driving convection. The helium burning shell is then indicated by the very pale line which appears to merge with the hydrogen burning shell after the second dredge up as the convective envelope deepens again. The periodicity seen in the helium burning luminosity at this point is due to the start of thermal pulses.

it is hot enough for helium to ignite. The triple- $\alpha$ process creates ${ }^{12} \mathrm{C}$ but it is also easy for ${ }^{12} \mathrm{C}$ to capture another ${ }^{4} \mathrm{He}$ nucleus to make ${ }^{16} \mathrm{O}$ at this point. Again the helium burning drives convection in the core which this time grows as the burning proceeds (Figure 1C). We now reach one of the currently contentious issues of stellar evolution. How much does the core grow? The region just outside the core is not fully convective in the usual sense but is stabilized by the composition change. It is not yet resolved how quickly the triple- $\alpha$ process can drive the outer edge through this gradient.

Eventually helium fuel is exhausted in the core too and helium burning moves to a shell that starts to follow the hydrogen-burning shell out through the envelope. During core helium burning the star had settled back to a lower luminosity, shrunk, and lost its deep convective envelope. It now moves to the asymptotic giant branch (AGB, Figure 1D). Like the hydrogen burning shell the helium burning shell heats up but now the extreme sensitivity to temperature means that the luminosity rises very quickly and forces the star to expand so much that the hydrogen burning shell is pushed out to colder parts and it goes out. This allows the convective envelope to reach in past it and almost down to the helium burning shell itself. The details of the interior structure of these stages are shown for a full stellar model in Figure 2. 


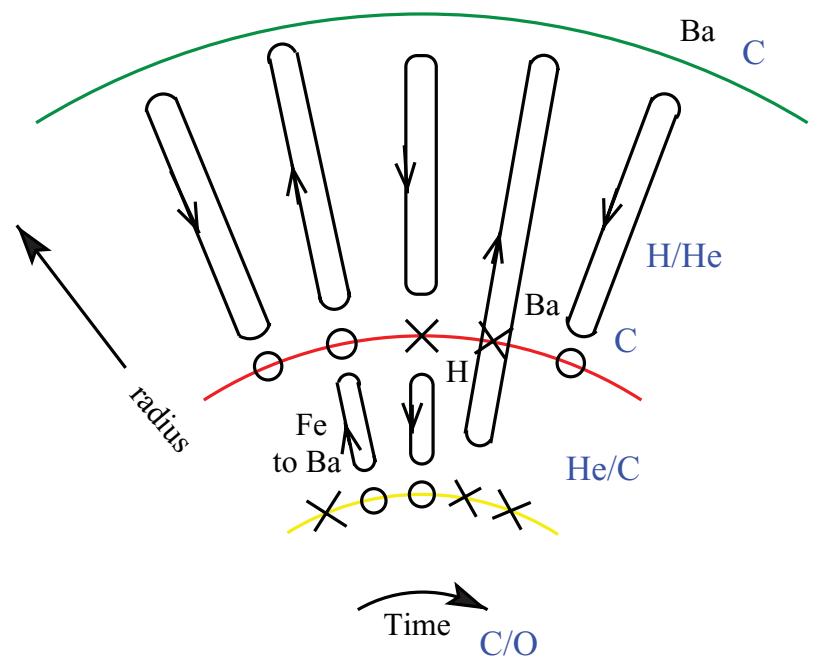

Figure 3. Schematic diagram of the thermally pulsing asymptotic giant branch. The innermost arc represents the He-burning shell, the next the H-burning shell, and the third the surface of the star. A circle indicates active burning while a cross indicates no or very quiescent burning. Time is measured clockwise. Loops are the extent of convective regions either between the two shells or throughout the $\mathrm{H}$-rich envelope. $\mathrm{Ba}$ is representative of the neutron capture isotopes created in the intershell region and dredged to the surface along with the ${ }^{12} \mathrm{C}$.

\section{Thermal Pulses}

At this point the two burning shells are separated by only $0.02 M_{\odot}$ of helium-rich material. The temperature sensitivity of the triple- $\alpha$ reaction and the thinness of the burning shell leads to an instability (Schwarzschild \& Härm 1965). Figure 3 shows schematically what happens and Figure 4 shows the detail for two pulses later in the evolution of the $5 M_{\odot}$ star shown in Figure 2. The instability sets in the He-burning shell. Its temperature rises unconstrained because it is so thin and the luminosity reaches as high as $10^{9} L_{\odot}$. This drives convection but only out to the still burning hydrogen shell. This convection rapidly mixes the products, the ${ }^{12} \mathrm{C}$, away from the burning region through the intershell region. It is whisked away so quickly that there is little opportunity for the capture of an additional ${ }^{4} \mathrm{He}$ nucleus to make any ${ }^{16} \mathrm{O}$. The star responds non-linearly eventually and expands: the instability is quelled but not before the hydrogen burning shell has cooled and extinguished. The convective envelope deepens and dredges into the intershell region that is rich in ${ }^{12} \mathrm{C}$ and carries it to the surface of the star. Eventually the hydrogen shell contracts and reignites until sufficient helium is added to the intershell region for the instability to set in again and the process repeats every $10^{4} \mathrm{yr}$ or so. This dredge up delays the core growth by pushing the hydrogen burning shell back down towards the helium burning shell. Often as much as $90 \%$ of the intershell region has its hydrogen replenished.

\section{Mass Loss}

Stars lose mass throughout their lives. The Sun is currently losing mass in the solar wind at a negligible rate of about $10^{-14} M_{\odot} \mathrm{yr}^{-1}$. The large radii and luminosities of giants makes it much easier to launch winds and so mass-loss rates increase dramatically. The precise mechanisms for launching and accelerating stellar winds are not well understood. For hot stars with radiative envelopes the winds are most likely radiation 


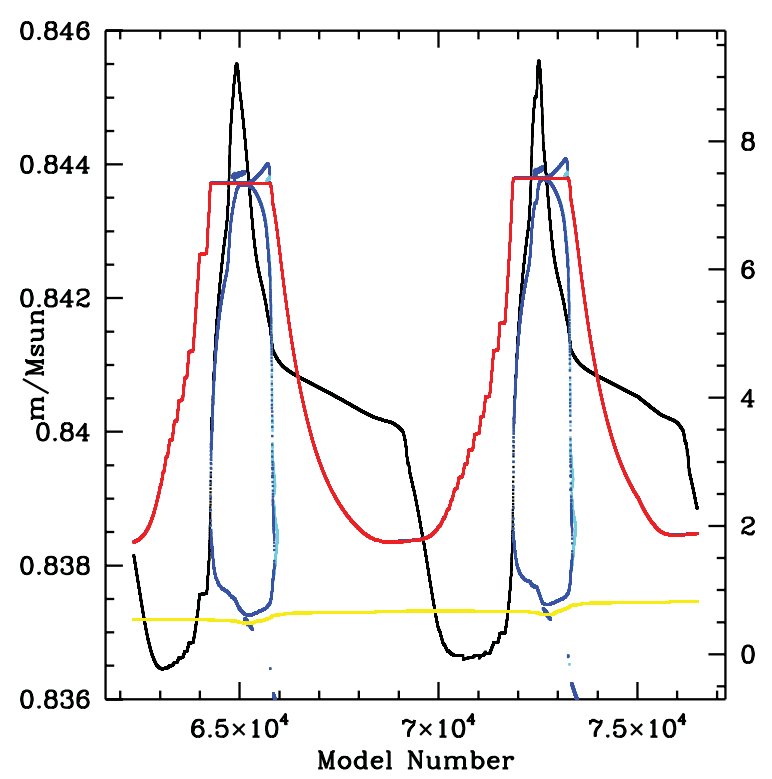

Figure 4. Detail of two thermal pulses later in the evolution of the $5 M_{\odot}$ star of Figure 2. Again the darkest line is $\log _{10}\left(L_{\mathrm{He}} / \mathrm{erg} \mathrm{s}^{-1}\right)$ according to the right-hand scale. The loops enclose the intershell convection during the peak of each pulse and the remaining darker line is the hydrogen burning shell at the base of the convective envelope. Note that most of the core growth before the pulse is wiped out by the dredge up.

driven. For cool stars, such as the Sun and red giants, the mechanism is much less certain but likely involves a magnetic dynamo operating in the convective envelope. Magnetic flux rises out of the stellar surface in loops and dissipates energy in a corona. This hot corona $\left(10^{6} \mathrm{~K}\right.$ in the Sun) can then drive a wind from the star. In very cool asymptotic giant branch stars and supergiants, stellar pulsation and the presence of dust, that can be accelerated by radiation pressure, can enhance mass loss considerably.

Various empirical mass-loss formulae have been derived but we must keep in mind that stars evolve on a very long timescale and so we are seeing only an instantaneous massloss rate rather than the long term average that we would really choose to apply to our models. We should also remember that the strongest winds are the easiest to measure. The most popular prescriptions today tend to have mass loss that increases very rapidly as a star approaches the very largest and most luminous phases of its evolution with most of its envelope being lost in a superwind towards the end of its life (Vassiliadis \& Wood 1993).

Thus the final stages of the evolution of an AGB star are a competition between core growth, as hydrogen in the envelope is burned to carbon and oxygen through the two burning shells, and loss of the envelope in a wind. When the mass loss is fast relative to the nuclear burning the carbon-oxygen white dwarf remnant is smaller in mass. At very low masses the lack of dust in any stellar wind reduces the mass loss rate. At the same time thermal pulses are weaker and lead to a smaller third dredge up. Both these changes conspire to allow the cores of low-metallicity stars to grow larger. At the lowest metallicities they can reach the point of cold carbon ignition and thermonuclear runaway before the envelope is lost (Lau, Stancliffe \& Tout 2008). For solar metallicity, the core only gets massive enough for carbon ignition in higher mass progenitors when the core is not very degenerate. 


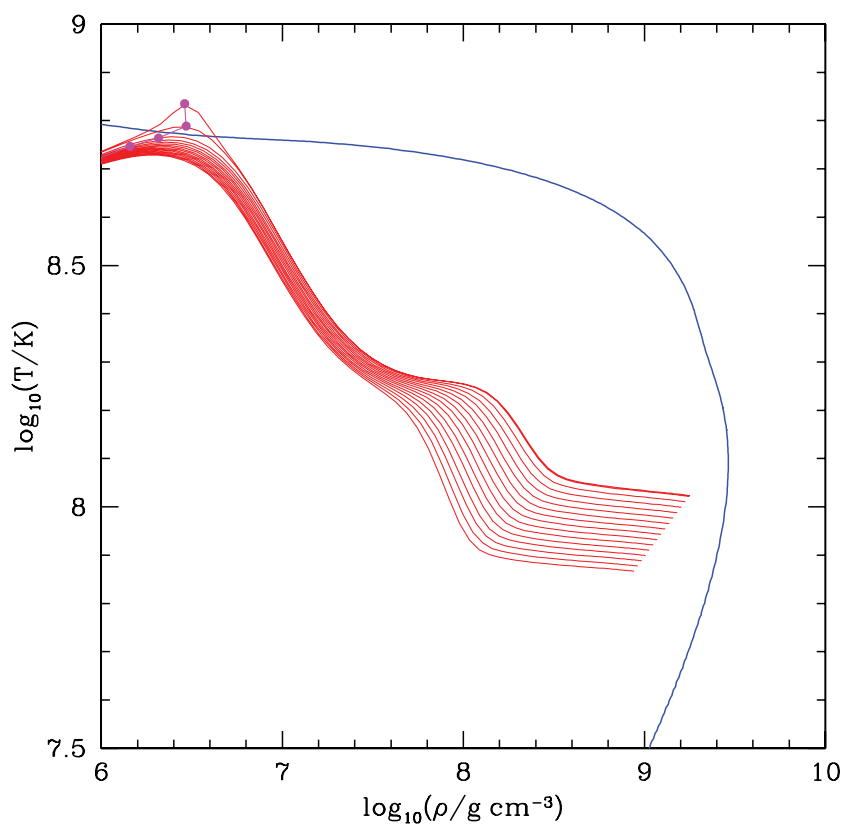

Figure 5. The line crossing both axes is the carbon ignition curve on which energy generation by carbon burning equals the energy loss in neutrinos. The other lines are the run of temperature and density through the degenerate core, at late times, increasingly later from bottom to top. In this case these cross the ignition curve at the center and a thermonuclear runaway can begin there under degenerate conditions. The rate of accretion of burnt material by the core is $3 \times 10^{-6} \mathrm{M}_{\odot} \mathrm{yr}^{-1}$. Ignition occurs close to the surface and carbon ignites under much less degenerate conditions. Grey filled circles indicate the path of the mass shell where carbon first ignites at mass $1.354 \mathrm{M}_{\odot}$ just $0.008 \mathrm{M}_{\odot}$ into the core.

\section{Carbon Burning}

In the most massive AGB stars carbon can ignite gently somewhere in the core. This occurs because the burning shells add material to the degenerate core fast enough to raise the degeneracy in the outer layers before ignition. The burning is then thermostatically controlled in the usual way. Burning is from the outside in because neutrino losses keep the inner, denser parts of the core cool. Figure 5 illustrates the ignition process. Core carbon burning leaves an oxygen-neon white dwarf if mass loss removes the envelope fast enough or an electron capture supernova if core growth wins.

\section{The Effects of a Binary Companion}

In the presence of a binary companion a star can only expand to fill its Roche lobe before material overflows. When this happens to a giant the process of mass loss tends to cause the giant to expand yet more. Unless earlier mass loss or transfer has inverted the mass ratio this leads to a positive feedback and common envelope evolution. There is also evidence that the mere presence of a companion can enhance the usual mass loss rate (Tout \& Eggleton 1988). This can both stabilize mass transfer by reducing the mass ratio but can also drive it. Even when the mass loss is steady, the giant envelope is generally lost much more quickly than from a single star (Tout \& Hall 1991). Many giants, including indeed most of those thought to be progenitors of the white dwarfs that go on to explode as SNe Ia, do not avoid the more catastrophic common envelope 


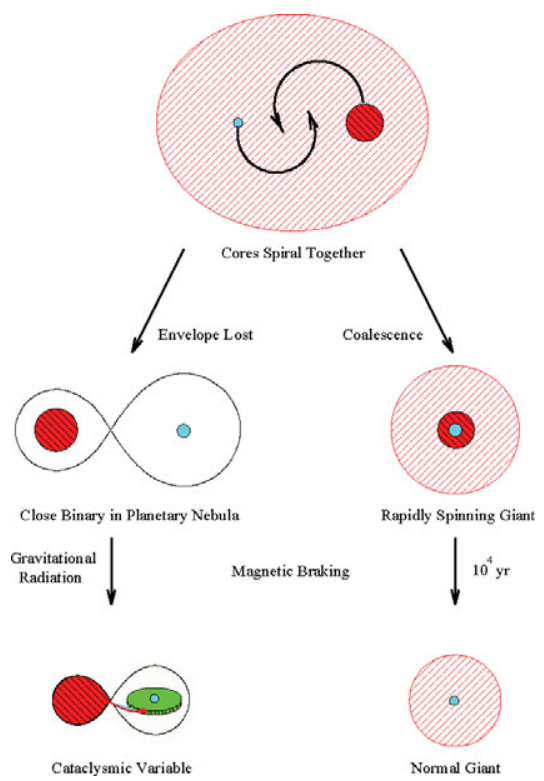

Figure 6. Common-envelope evolution. After dynamical mass transfer from a giant, a common envelope enshrouds the relatively dense companion and the core of the original giant. These two spiral together as their orbital energy is transferred to the envelope until either the entire envelope is lost or they coalesce. In the former case a close white-dwarf and main-sequence binary is left, initially as the core of a planetary nebula. Magnetic braking or gravitational radiation may shrink the orbit and create a cataclysmic variable. Coalescence results in a rapidly rotating giant which will very quickly spin down by magnetic braking.

evolution (Paczyński 1976) illustrated in Figure 6. This leads to rapid mass loss and orbital shrinkage.

Thus it would appear that binary interaction tends to give mass loss the advantage over core growth. However this is not always the case because when it occurs at the right time the thermal pulses that slow down core growth can be avoided. Figure 7 shows an example of a $7 M_{\odot}$ star in which rapid mass loss has been applied before the second dredge up is complete. This means that the hydrogen and helium burning shells never get close enough to become unstable to thermal pulses before all the hydrogen has been lost. Without thermal pulses to hamper core growth a carbon oxygen core can then grow to its ignition mass within just a helium envelope. The thermonuclear runaway would then lead to an explosion in the absence of hydrogen that would look very much like an SN Ia. Such a process was discussed by Iben and Tutukov (1985). It is ostensibly included in population synthesis models such as those of Hurley, Tout and Pols (2002) but occurs only very rarely. However, as we have discussed, our understanding of mass loss even from single stars is very incomplete and much of binary star evolution, particularly common envelope evolution, remains guesswork. It is quite possible that the physics of stars may well conspire to make these stars an important contributor to SNe Ia. They might even leave no detectable remnant.

\section{Conclusions}

The carbon-oxygen white dwarfs, which are recognized as the immediate progenitors of SNe Ia are born as the cores of AGB stars. The evolution of the late stages of AGB star evolution is a competition between core growth by nuclear fusion and envelope loss 


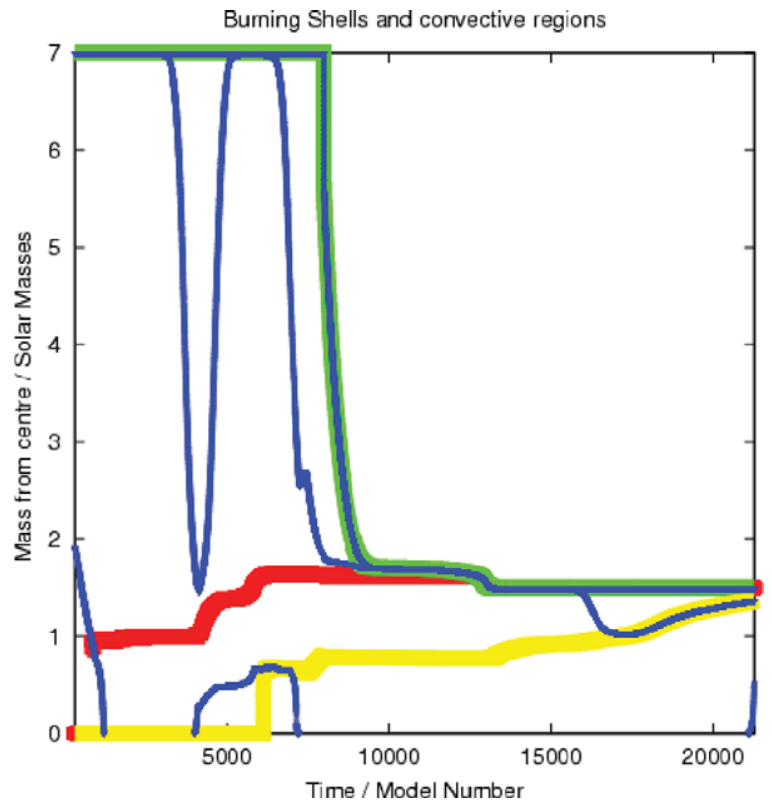

Figure 7. The internal evolution of a $7 M_{\odot}$ star truncated so that thermal pulses are prevented and the core can grow to the point of central carbon ignition.

in a stellar wind. Binary interaction can lead to both lower mass cores and unusually high mass cores, and even to progenitors very different from those considered standard today.

CAT is very grateful to Churchill College Cambridge for his Fellowship and to the STFC for funding his attendance at this symposium.

\section{References}

Hurley, J. R., Tout, C. A., \& Pols, O. R. 2002, MNRAS, 329, 897

Iben, I. Jr. \& Tutukov, A. V. 1985, ApJS, 58, 661

Lau, H. H. B., Stancliffe, R. J., \& Tout, C. A. 2008, MNRAS, 385, 301

Paczyński, B. 1976, in: P. P. Eggleton, S. Mitton S. \& J. Whelan (eds), Structure and Evolution of Close Binary Systems, Proc. IAU Symposium No. 73, (Dordrecht: Reidel), p. 75

Schwarzschild, M. \& Härm, R. 1965, ApJ, 142, 855

Tout, C. A. \& Eggleton, P. P. 1988, MNRAS, 231, 823

Tout, C. A. \& Hall, D. S. 1991, MNRAS, 253, 9

Vassiliadis, E. \& Wood, P. R. 1993, ApJ, 413, 641 